\title{
Caracterização de idosos residentes no município de Colméia - TO
}

\author{
Characterization of elderly residents in the municipality of Colméia-TO
}

Matheus da Silva Barbosaㄹ, Wanclecio Rodrigues Rocha², Roberta Lopes de Queiroz $z^{3}$, Adriana Keila Dias ${ }^{4}$, Glaucya Wanderley Santos Markus 5 , Reobbe Aguiar Pereira ${ }^{6}$, Giullia Bianca Ferraciolli do Couto $^{7}$

\section{RESUMO}

O envelhecer é um acontecimento que estará presente na fase de vida do homem evidenciado pela redução graduada da função fisiológica, ocasionado por alterações psicológicas, físicas e socioeconômicas. Este estudo tem objetivo em descrever a caracterização de idosos residentes no município de Colméia - TO. Pesquisa de campo de abordagem qualiquantitativa, exploratório e descritivo. A população deste estudo foi composta por 30 (trinta) idosos no mínimo 60 anos de idade, e que faça parte do quadro de idosos do município de Colméia - TO. Para verificar o perfil característicos dos idosos da região pesquisa foi efetivada uma análise exploratória das variáveis idade, sexo, nível de escolaridade, estado civil, números de filhos, considerações sobre a saúde, doenças crônicas e a importância da sexualidade. No fator idade, $60 \%$ dos idosos tem entre 60 e 70 anos, $30 \%$ tem entre 71 a 80 anos de idade e $10 \%$ tem entre 81 a 90 anos. Grau de escolaridade $50 \%$ não são alfabetizados e apenas $4 \%$ concluiu ensino superior. Nesse sentido, os assuntos relacionados ao envelhecimento populacional estão envolvidos no contesto social, alinhados aos sistemas de saúdes e de assistência social prestada a esse grupo. Foi possível verificar que a qualidade de vida e o envelhecimento saudável dá-se como uma abrangência que corresponde a uma junção de causas que se relaciona a vivencia diária do idoso.

Palavras-chave: Envelhecimento. Saúde. Patologias.

\section{ABSTRACT}

Aging is an event that will be present in the man's life stage, evidenced by the gradual reduction of physiological function, caused by psychological, physical and socioeconomic changes. This study aims to describe the characterization of elderly residents in the municipality of Colméia - TO. Field research with a qualitative, exploratory and descriptive approach. The population of this study was composed of 30 (thirty) elderly people with at least 60 years of age, and who are part of the elderly in the municipality of Colméia-TO. To verify the characteristic profile of the elderly in the research region, an exploratory analysis of the variables age, sex, education level, marital status, number of children, health considerations, chronic diseases and the importance of sexuality was carried out. In the age factor, $60 \%$ of the elderly are between 60 and 70 years old, $30 \%$ are between 71 and 80 years old and $10 \%$ are between 81 and 90 years old. Education level $50 \%$ are illiterate and only $4 \%$ have completed higher education. In this sense, issues related to population aging are involved in social contests, in line with the health and social assistance systems provided to this group. It was possible to verify that the quality of life and healthy aging occurs as a scope that corresponds to a combination of causes that is related to the daily experience of the elderly.

Key Words: Aging. Health. Pathologies.
${ }^{1}$ Graduado em Enfermagem na Faculdade Guaraí-FAG. E-mail: matheus071998@outlook.com

${ }^{2}$ Graduado em Enfermagem na Faculdade Guaraí-FAG. E-mail: wancleciorr21@gmail.com

${ }^{3}$ Graduanda em Enfermagem na Faculdade Guaraí-FAG. E-mail: roberta.queiroz15@gmail.com

${ }^{4}$ Enfermeira. Mestra em Ciências Ambientais. Docente da Faculdade Guaraí-FAG. E-mail: adrianakeiladias@hotmail.com

${ }^{5}$ Enfermira. Mestra em Bioengenharia com Ênfase em Saúde. Docente da Faculdade Guaraí-FAG. E-mail: glaucyamarkus@outlook.com

${ }^{6}$ Enfermeiro. Mestre em Ciências Ambientais. enfreobbe@gmail.com

${ }^{7}$ Enfermeira. Mestra em Bioengenharia com Ênfase em Saúde. Docente da Faculdade Guarai-FAG. E-mail: giulliabianca@hotmail.com 
DOI: 10.18606/2318-1419/amazonia.sci.health.v9n2p2-16 Revista Amazônia Science \& Health
BARBOSA, MS. ROCHA, WR. QUEROZ, RL. DIAS, AK. MARKUS, GWS. PEREIRA, RA. COUTO, GBF.

Caracterização de idosos residentes no município de Colméia - TO

\section{INTRODUCCÁO}

De acordo com a Organização Mundial da Saúde (OMS), idoso são pessoas que contenham 60 anos ou mais. O Brasil tem mais de 28 milhões de pessoas nessa faixa etária, número que representa $13 \%$ da população do país. E esse percentual tende a dobrar nas próximas décadas, segundo a Projeção da População, divulgada em 2018 pelo IBGE. No Tocantins a população de pessoas com 60 anos ou mais até o último trimestre de 2019 era de 228 mil. A porcentagem em relação à população total do Tocantins (pouco mais de 1,5 milhões) é de $14,6 \%$. Os números são da pesquisa nacional por amostra de domicílios trimestral contínua do Instituto Brasileiro de Geografia e Estatística (IBGE). O envelhecer trata-se de um trajeto gradual que acontece naturalmente e afeta todo o corpo fisiologicamente, causando mudanças biológicas no ser humano, decorrente de causas envolvendo o meio social, cultural e psíquicos. $O$ envelhecimento compreende particularidades com mudanças que alteram aparências corriqueiras e saudáveis de uma pessoa, acarretando para inovações e novas perspectivas de viver. ${ }^{1}$ Entende-se que idosos em países em aditamento são pessoas com idade acima de 60 anos e em países desenvolvidos, a partir de 65 anos de idade. ${ }^{2}$

O aumento de idosos no mundo se aproxima de $3 \%$ anualmente, é provável que, no ano de 2050, 2,1 bilhões de pessoas serão idosos. Presentemente, sabe-se que 962 milhões de indivíduos tem 60 anos de idade ou mais, correspondendo a $13 \%$ da população mundial. Em 2050, é provável que todos os lugares do mundo, com exceção da África, vão estar nessa media. Além disso, o Brasil, compreende a $13 \%$ de pessoas com mais de 60 anos de idade. Além do mais esses dados poderão chegar a 29,3\% a partir de $2050 .{ }^{3}$

O envelhecer é um acontecimento que estará presente na fase de vida do homem evidenciado pela redução graduada da função fisiológica, ocasionado por alterações psicológicas, físicas e socioeconômicas podendo ser modificada de um sujeito para outro, sendo pouco a pouco para determinados indivíduos e de forma rápida para outros. No entanto isto não está condicionado exclusivamente a categoria hereditária, e sim, sobretudo, aos estilos que apresentamos no decorrer da vida, tais quais os hábitos alimentares e a prática de atividade física. Hábitos impróprios e até mesmo falta de movimentação, exercícios poderão colaborar gradativamente para acrescentar no aumento de patologias crônicas e inabilidades. ${ }^{2}$ 
Os costumes de praticar exercícios físicos promovem a terceira idade uma vida saudável, preservando autonomia e liberdade para tarefas cotidianas, resultando em independência prolongada. Mostrar-se importância diante do declínio de alvos contraproducentes causados pelo envelhecimento nos artifícios biológicos e psicológicos tornando mínimos os efeitos contrários que provocam estricção, abatimento e danos a funcionalidade. Os benefícios das atividades físicas para a população da terceira idade em academias cooperam de forma positiva para a qualidade de vida, liberdade e contentamento e atividades de trabalho dos mesmos. Entende-se que os níveis de qualidade de vida podem ser influenciados pela atmosfera global, sendo imprescindível que nem todos os exteriores de vida são acarretados no exercício de atividades que oferecem bem-estar, porém é uma formidável ferramenta geradora de auto estima funcional e saúde aos idosos. ${ }^{4}$

Assim sendo, o objetivo geral do presente trabalho é descrever a caracterização de idosos residentes no município de Colméia - Tocantins. E tendo como objetivos específicos, observar os atributos sociais e anatômicos de bem-estar dos idosos, identificar o perfil quanto a prática de atividade física, verificar o perfil epidemiológico, dos participantes, de acordo com doenças crônicas e analisar a prevalência de atividade sexual ativa na população do município citado.

\section{MATERIAL E METODOS}

Este estudo é uma pesquisa de campo de abordagem quantitativa e quantitativa, exploratório-descritivo. O prosseguimento do estudo atendeu às normas nacionais e internacionais de ética em pesquisa envolvendo seres humanos, resguardados pela a Resolução o 466, de 12 de outubro de 2012 do Conselho Nacional de Saúde (CNS). A pesquisa obteve a aprovação do Comitê de Ética em Pesquisa da Universidade Estadual do Tocantins - UNITINS, campus de Palmas-TO, emitido sob parecer № 4.296.422.

A população deste estudo é composta por 30 (trinta) idosos com mínimo 60 anos de idade, e que faça parte do quadro de idosos do município de Colméia-TO. Os critérios de inclusão idosos que fazem parte do município de Colméia, ambos o sexo, e concordou em participar do estudo e assinou o Termo de Consentimento Livre e Esclarecido (TCLE). Não participaram da pesquisa idosos não cadastrados no Centro de Convivência de Colméia, Serviço Convivência e fortalecimento de vinculo (SCVF), que apresentem comorbidades 
que impeçam a fala e o entendimento das questões da entrevista, e que não aceitarem assinar o TCLE.

A pesquisa foi realizada no município de Colméia-TO. A seleção dos (a) participantes da pesquisa seguiu-se de forma aleatória e a nível domiciliar, respeitando o protocolo de biossegurança do COVID-19. Todos os participantes foram esclarecidos sobre as finalidades e os objetivos da pesquisa e sobre a interrupção da pesquisa instantaneamente, caso alguma sentisse indícios de incomodidade e/ou desejasse desistir de participar do estudo antes de assinarem o Termo de Consentimento Livre e Esclarecido.

A coleta de dados foi realizada do dia 24 a 30 de setembro de 2020 através de um questionário, preenchido pelos os pesquisadores responsáveis. No mesmo, havia um total de 17 perguntas, sendo todas objetivas. O formulário foi dividido em duas partes sendo que a primeira continha data e assinatura do entrevistado ou responsável pelo mesmo e na segunda continha dados sobre idade, profissão e escolaridade, quantidade de filhos, medicações utilizadas e a importância da sexualidade na vida do idoso.

\section{RESULTADOS E DISCUSSÃO}

A forma como se envelhece é também influenciada pela sociedade, comunidade e família. ${ }^{5}$ Relacionado a esta definição está a ideia de que para além dos cuidados com a saúde física, existem outros fatores que influenciam o modo como os indivíduos e a população envelhecem. ${ }^{6}$ Circunstâncias externas ao sujeito, e ele próprio, determinariam o grau de bem-estar, satisfação ou felicidade. Uma vez que a conceptualização da qualidade de vida é, simultaneamente, social e individual, fatores como a idade, sexo, nível de escolaridade, condição sócio ocupacional e localização, entre outros, influenciam significativamente o esquema conceptual de cada sujeito, podendo-se falar de uma qualidade de vida subjetiva.

"A saúde afeta a vida, quando se pensa na vida plena, tem-se em primeiro lugar na mente uma vida com saúde". ${ }^{7}$ A saúde surge constantemente como um dos aspetos mais importantes na vida das pessoas, nomeadamente nos idosos e é uma das variáveis determinantes da satisfação da vida. O autor ainda sustenta a ideia de que "as vulnerabilidades da idade avançada podem ser mais de natureza psicológica do que física".

Deste modo, a compreensão do envelhecimento saudável, segundo a definição da OMS, é abrangente e relevante para todas as pessoas idosas, mesmo para aquelas que 
convivem com a experiência de doenças crônicas; também não está centrada na ausência de agravos e nem tampouco restrita à funcionalidade do idoso, mas em um processo que possibilitará a construção de habilidades que Ihe permitirão vivenciar o envelhecimento da melhor forma possível. ${ }^{8}$ Nesse contexto, para verificar o perfil característicos dos idosos da região pesquisa foi efetivada uma análise exploratória das variáveis idade, sexo, nível de escolaridade, estado civil, números de filhos, considerações sobre a saúde, doenças crônicas e a importância da sexualidade.

Gráfico 01: Composição da população residente em Colmeia - TO, por sexo.

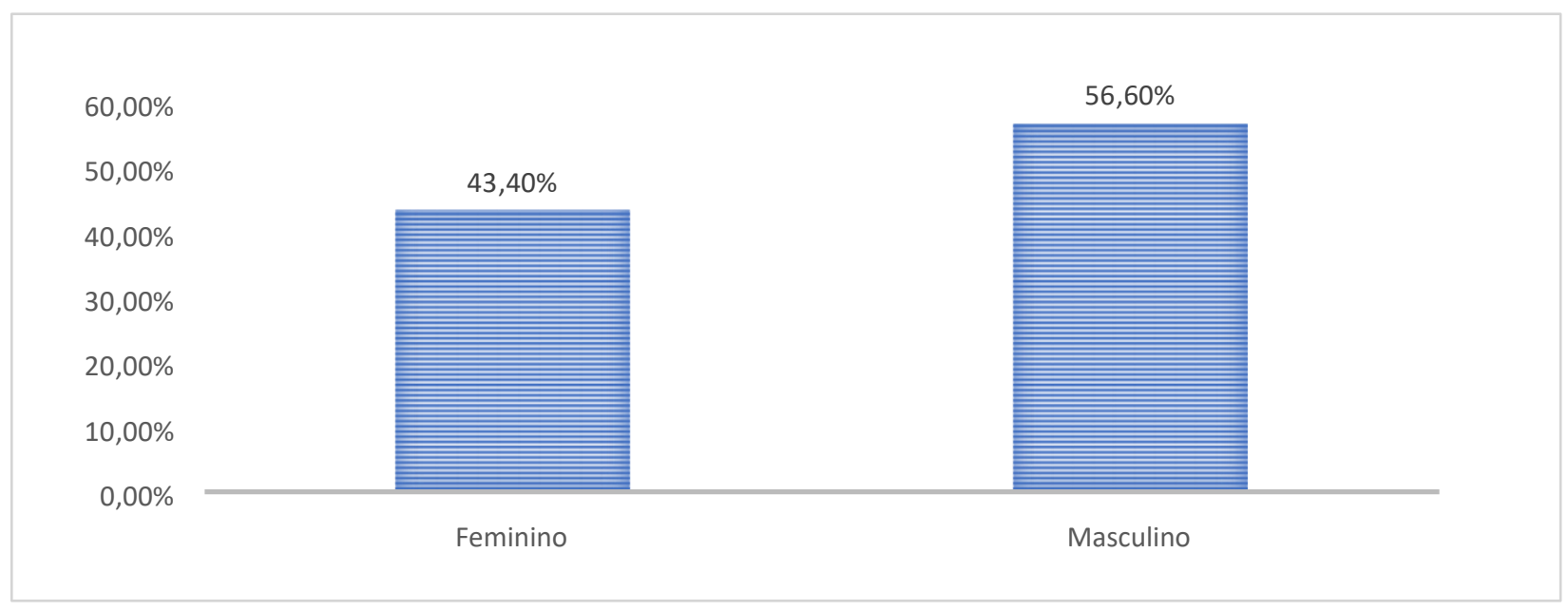

Fonte: Elaborado pelos autores, 2020.

\section{Sexo}

Em 1996, dos 12,4 milhões de idosos 54,4\% eram do sexo feminino. Compreendese que o alto tributo é crescente em relação ao sexo masculino. A duração populacional de mulheres esclarece essas intercalações na composição por sexo. Em decorrência disso, quanto mais antigo é o eventual analisado mais grande é a dimensão do sexo feminino. A auto índice de mulheres na terceira idade apresenta consequências formidáveis levando em consideração os processos relacionados as políticas públicas. Isso diz respeito ao fato de que o sexo feminino pode viver mais tempo que o sexo masculino, porem as mulheres estão mais sujeitas a apresentarem mais chances de desenvolver determinadas patologias em relação aos homens. Além do mais o aumento dimensional do sexo feminino residindo só correspondeu a $14 \%$ em 1998 . Nessa perspectiva, compreende-se que $12,1 \%$ reside com a família ou com outros parentescos. No ano de 1995, maior parte das mulheres residentes com familiares $74 \%$ eram viúvas. Observando-se que maior parte delas não 
adquiriu conhecimento formal no mercado trabalhista, não obteve boa educação, motivo pelo qual requerem auxílio especializado da família das autoridades estaduais. ${ }^{9}$

Quando se compara os dados obtidos nessa pesquisa com os dados nacionais, notase ao contrário pois $56.6 \%$ são do sexo masculino, isso implicaria em um estudo para saber as causas contrário ao restante do país. Como resultado de uma desigualdade de gênero na expectativa de vida, existe essa proporção maior de mulheres do que de homens nesse grupo populacional. As mulheres vivem, em média, sete anos mais do que os homens e estão vivendo mais do que nunca. Outra característica deste grupo populacional é que existe uma maior proporção de viúvas do que em qualquer outra faixa etária. Uma razão que poderia explicar essa situação é que, por tradição, a mulher tende a se casar com homens mais velhos do que ela, o que, associado a uma mortalidade masculina maior do que a feminina, aumenta a probabilidade de sobrevivência da mulher em relação ao seu cônjuge.

Os dados encontrados e discordantes poderão nos levar as várias vertentes: estaria os homens cuidados mais da parte de promoção e prevenção do que as mulheres? Ministério da saúde investe no programa saúde do homem para fazer a inserção dessa população que não tem esses hábitos de prevenção das doenças. A outra vertente suposta é as pesquisas gerontológicas mostram que as mulheres de idade avançada (e não os homens) detém taxas mais altas de institucionalização, seria um motivo pelo qual a pesquisa encontrou na amostra por domicilio mais homens?

Gráfico 02: Variável relacionado a idade dos participantes da pesquisa.

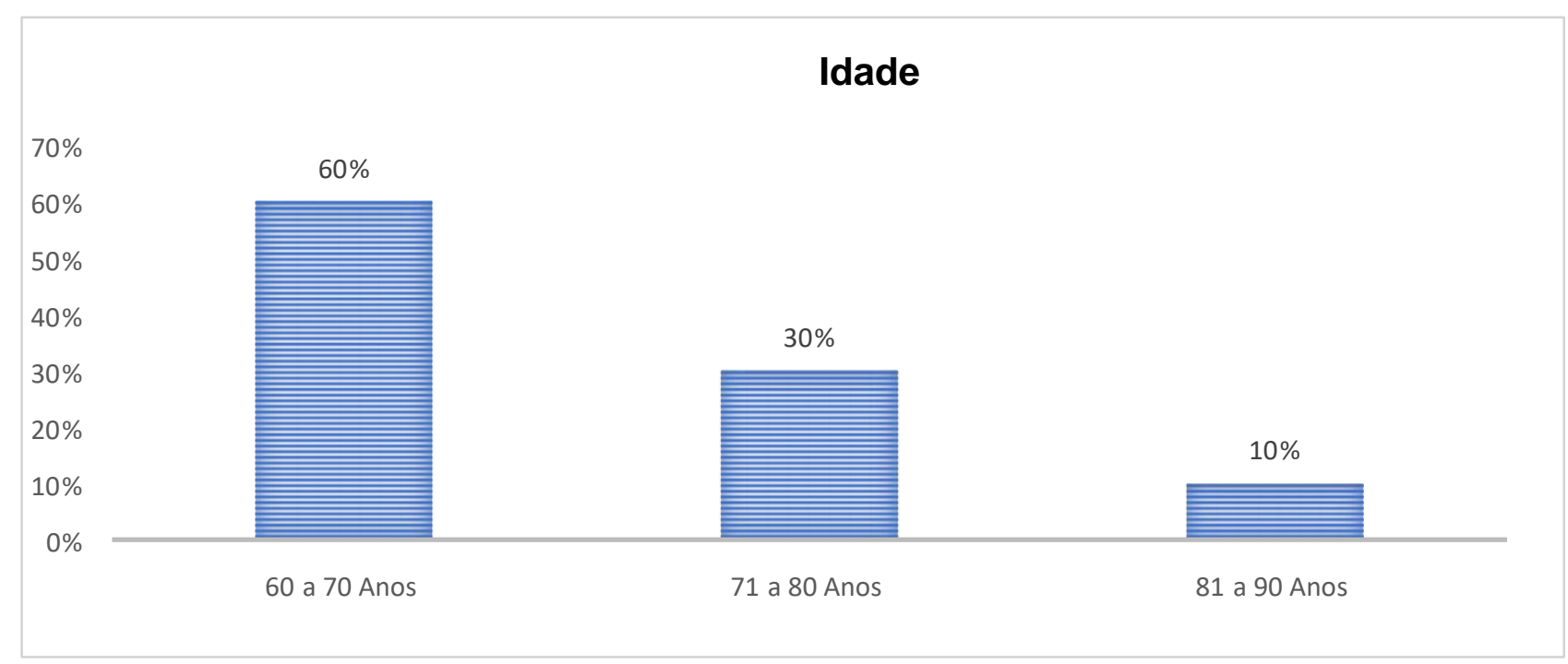

Fonte: Elaborado pelos autores, 2020. 
É bastante utilizado o critério denominado por idade cronológica para a definição do envelhecimento, embora o mesmo seja considerado um critério arbitrário. Ainda que o envelhecimento seja um processo natural e universal, que respeita a todos e quaisquer seres vivos, a forma como cada indivíduo envelhece é própria e única, o que torna esse processo heterogéneo. Sendo que indivíduos com a mesma idade cronológica podem estar em diferentes fases de envelhecimento ${ }^{10}$, assim o processo de envelhecimento deve ser entendido como um processo natural, dinâmico, progressivo e irreversível que nos acompanha desde o nascimento até à morte. ${ }^{11}$

A OMS menciona, idosos de 60 a 74 anos, anciãos de 75 a 89 anos e velhice extrema, 90 anos ou mais. No Brasil, de acordo com o Instituto de Pesquisa Econômica Aplicada (IPEA), são estimados idosos jovens os que tem 60 e 70 anos de idade; medianamente idosos a partir de 70 até 80 anos; e muito idosos acima de 80. A demais, existe um grande número de idosos com idade maior ou igual a 80 anos e ainda, maior ou igual a 85 anos. ${ }^{12}$

Hoje em dia, os estudos acerca de envelhecer mencionam a existência de três categorias de indivíduos com idade mais avançada: os idosos jovens, os idosos velhos e os idosos mais velhos. De 65 a 74 anos considera-se como idoso jovem, que ainda tem vida produtiva. De 75 a 84 anos, são considerados como idosos velhos, de 85 anos ou mais os mais velhos, ou seja, apresentam probabilidade maior de fraquezas surgimento de doenças, e maior dificuldade de desempenhar funções. ${ }^{13}$ Conquanto a categorização consistir bastante e habitual, as pesquisas a cada vez mais mostrar-se que o processo de envelhece é uma experiência heterogênea, convivência como um conhecimento particular. ${ }^{14}$

A causa favorável consistir em fatores em que se apresentam a "detonação demográfica do envelhecimento no Brasil apesar que não se caracteriza por crescente do número de "idosos muito velhos". Avaliar-se que a dimensão de maiores de 80 anos entre os idosos varia de $9 \%$ a $11,3 \%$ dentre 1995 e 2020, apesar que o aumento daqueles acima de 70 anos chegue acrescentando progressivamente.

Dentro desse contexto observa-se através dos dados obtidos nessa pesquisa que maior parte dos idosos entrevistados (60 a 70 anos de idade) correspondem a $60 \%$ do resultado, enquanto que apenas $10 \%$ tem idade entre 81 e 90 anos, um fator importante 
pois o resultado predominante pode estar relacionado com qualidade de vida desses idosos tendo em vista que uma minoria alcança idade superior ou igual a 90 anos.

Gráfico 03: Situação conjugal das pessoas de 60 anos ou mais pesquisadas.

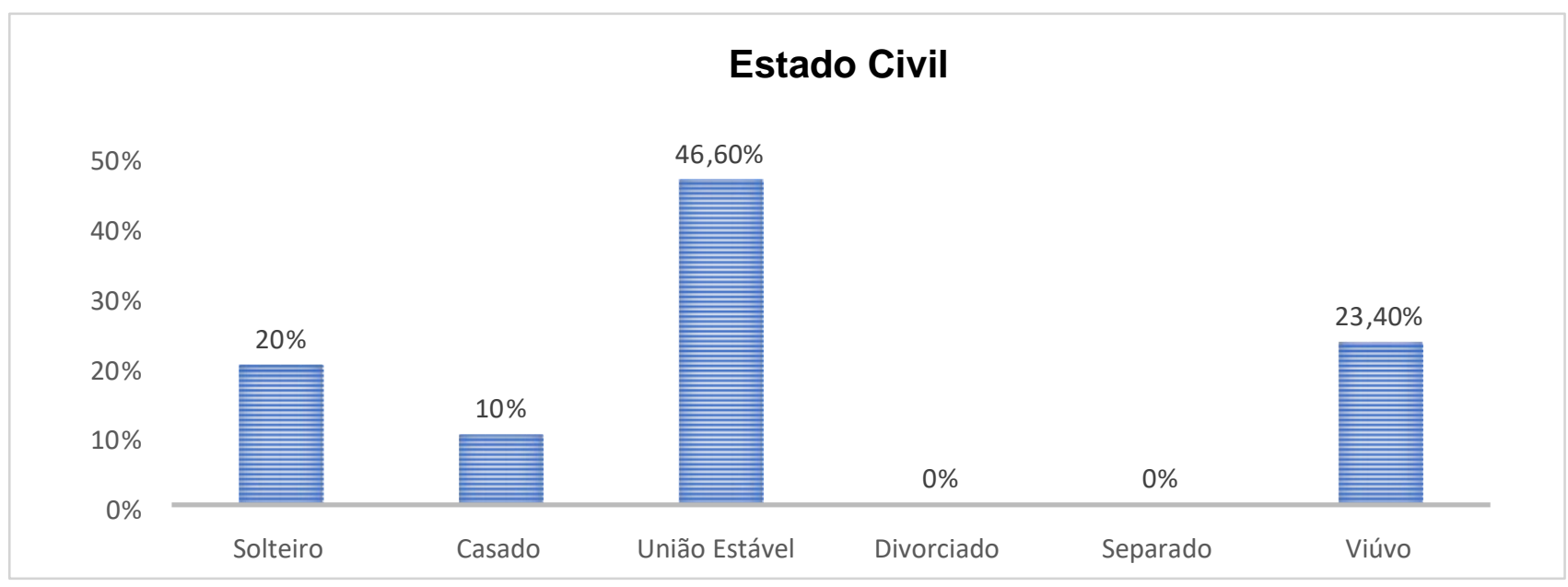

Fonte: Elaborado pelos autores, 2020.

A distribuição das pessoas por estado conjugal e estado civil foi levantada na PNAD (Pesquisa Nacional Por Amostra de Domicílio) a partir de 2011, permitindo analisar o comportamento desta variável ao longo do tempo. Na população de 15 anos ou mais de idade, a proporção que vivia em união estável foi de $56,3 \%$, em 2015 , sendo que aquelas pessoas que eram casadas no civil e/ou religioso eram $36,5 \%$ e as unidas de forma consensual $19,8 \%$.

Segundo os dados que emergiram da pesquisa de idosos, a união estável acumulou $46,6 \%$, seguido de viúvo que a barganhou $23,4 \%$ a situação solteira ficou no ranking da $3^{\text {a }}$ posição com um total de $20 \%$, e somente $10 \%$ da amostra e casado, sendo que divorciado e separado não apareceram nos dados. ${ }^{15}$

No que se refere ao número de filhos, na pesquisa foi utilizado a variável relacionado ao número de filhos onde emergiram as seguintes categorias: 0 a 2 filhos $36.6 \%$, 3 a 5 ficou com $36.6 \%$, enquanto 6 a 8 ficou com 20\%, e não menos importante de 9 a 10 a barganhou $6,8 \%$.

Assim levando em consideração a trajetória da fertilidade e seu início em meados dos anos de 1960 observa-se que o número médio de filhos por caiu de 6 para menos de 2 filhos por mulher. As mudanças na produtividade, incidiu de tal maneira no Brasil assim como mundialmente. Porem no brasil o índice de redução foi maior em relação à média 
mundial. Além do mais, os dados encontrados na variável pesquisada e concordante com a literatura brasileira e no mundo, a parcela da população com idade acima de sessenta anos está crescendo em um ritmo mais acelerado do que qualquer outro grupo etário. Historicamente, o número de crianças sempre foi superior ao número de idosos. Porém, espera-se que em 2050 o percentual da população mundial acima de sessenta anos ultrapasse o percentual de jovens de até 14 anos. No Brasil, essa transição deve ocorrer já em $2030 .^{16}$

Gráfico 04: Avaliação do nível de escolaridade dos participantes.

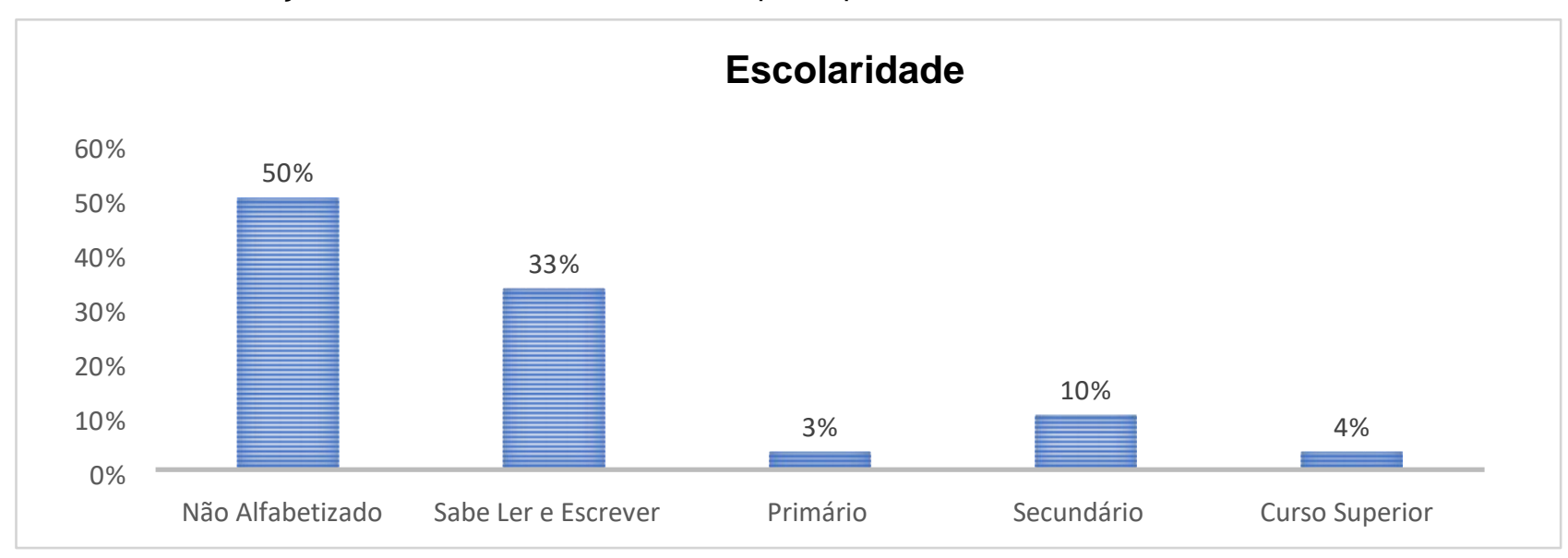

Fonte: Elaborado pelos autores, 2020.

Sabe-se que no brasil o analfabetismo ainda é um fator alarmante, e isto pode ser observado no grupo de idosos inseridos na pesquisa onde nota-se que $50 \%$ não são alfabetizados e um mínimo de $4 \%$ possui curso superior.

O baixo nível de alfabetização dos idosos pode ser devido as maneiras associadas a qualidade de saúde, condição socioeconômica. ${ }^{15}$ Destaca-se como atributos de envelhecer com qualidade, o envolvimento do idoso com equipamentos tecnológicos e a competência de poder se situar de forma positiva, tendo sua espiritualidade e conhecimento acerca de seu modo de viver", porém o analfabetismo acaba causando dificuldades nesse processo. ${ }^{17}$

A maioria da população não-alfabetizada no Brasil é composta por pessoas de idade mais avançada, ou seja, idosos e adultos mais velhos, principalmente as mulheres, os negros e afrodescendentes, os indígenas e os residentes nas áreas rurais e na região Nordeste. Conhecemos que existem escolas destinadas às crianças, aos jovens e, até mesmo, aos adultos trabalhadores (da indústria), assim como é o caso da EJA. Mas, por 
outro lado, não há escolas propostas aos idosos, e que acatem especificamente às suas precisões e as particularidades enquanto sujeitos sociais. ${ }^{18}$

O enigma do analfabetismo que arremete as populações mais idosas tem analogia direta com o fato de ser este um grupo social deixado pelo Poder Público quanto a declaração de políticas educacionais. $O$ fenômeno da exclusão educacional da população idosa nos leva a compreender o sistema educacional como uma estrutura burocrática criada com o fim de promover a formação e a qualificação da mão-de-obra jovem para o mercado de trabalho. Assim, como se sabe, a exclusão dos idosos do mercado de trabalho prédeterminaria a sua eliminação do campo da educação.

Além do mais sabe-se que no Brasil, até a década de 50 do século passado, antes de o país apresentar forte crescimento industrial, a leitura e a escrita eram pouco usadas pela maioria da população. Ler e escrever eram atividades que se resumiam à assinatura de um documento e à elaboração de uma carta. Essa situação se alterou profundamente. Basta caminhar pelas ruas de uma cidade, lotadas de textos escritos, ou entrar em uma Unidade Básica de Saúde, com vários cartazes pendurados pelas paredes, para verificar que, hoje, quem não tem acesso à linguagem escrita está à margem da estrutura social vigente, a qual privilegia tecnologias da informação e da informatização. ${ }^{18}$

Gráfico 05: Características das doenças crônicas em idosos de Colméia - TO.

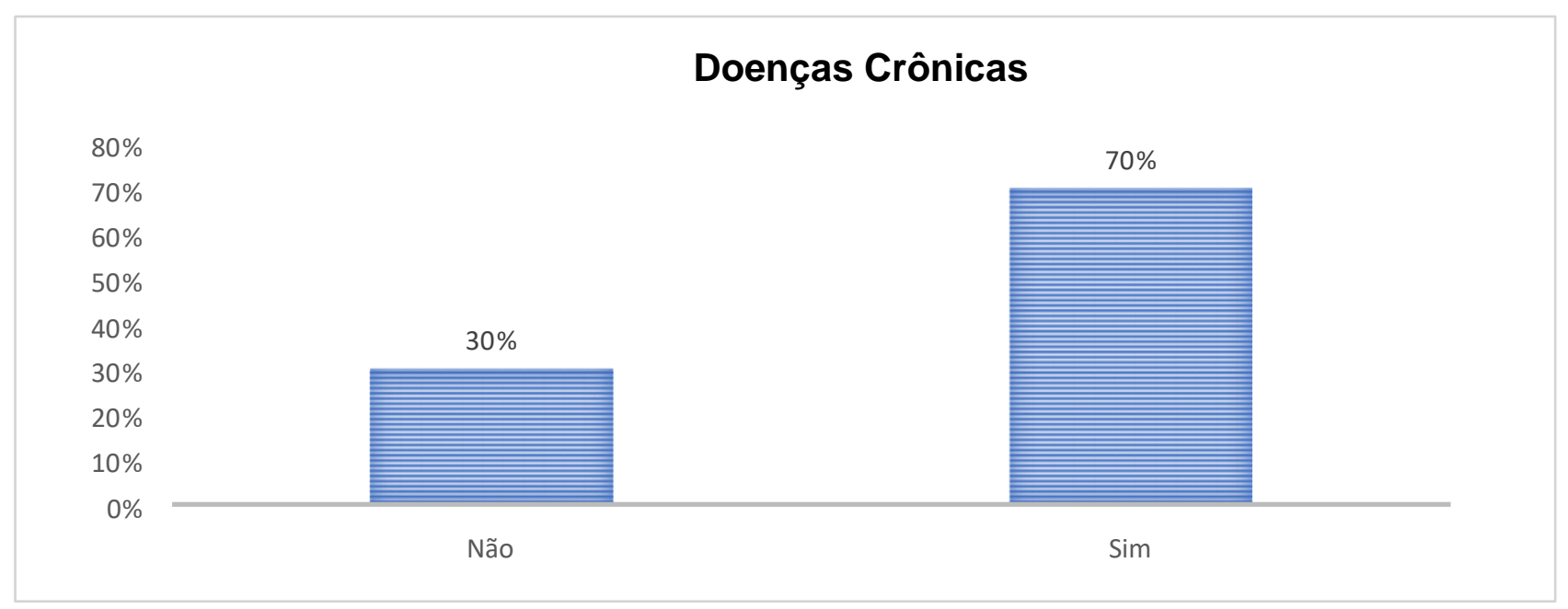

Fonte: Elaborado pelos autores, 2020. 
Observa-se através do gráfico uma grande porcentagem de idosos (70\%) afirmam conviver com algum tipo de doença crônica.

Tal crescimento dessas enfermidades que não podem ser transmitidas, confirmam os maiores motivos de causas de morte mundialmente. Salvo que as doenças crônicas causam 38 milhões de mortes por ano, levando em consideração que $3 / 4$ desse total acometem países como Brasil de renda baixa, ademais, destaca-se crescente aumento de mortalidade antecedente aos 70 anos de idade devido a limitações encontradas. ${ }^{19}$

A grande mortalidade e inabilidades atualmente se dão devido as doenças crônicas. Dentre estas a que mais afetam a terceira idade são Hipertensão Arterial Sistêmica e Diabetes Mellitus, que estão relacionadas ao aumento patológico de doenças cardiovasculares e cerebrais, causando maiores índices de morte..$^{20}$

O empenho da capacidade funcional do idoso tem efeitos importantes para a família, a comunidade, para o sistema de saúde e para a vida do próprio idoso, uma vez que a incapacidade traz maior vulnerabilidade e dependência na velhice, fornecendo para a diminuição do bem estar e da qualidade de vida dos idosos. Incluir os fatores que contribuem para a incapacidade ligada do idoso pode auxiliar o planejamento de políticas públicas na organização de táticas preventivas mais eficazes que irão, consequentemente, diminuir os custos com o serviço de saúde e tornar mínimo a carga sobre a família.

Os resultados deste estudo demonstraram que as doenças crônicas apresentam uma forte influência na capacidade funcional do idoso. A presença de hipertensão arterial aumentou significante nas últimas décadas seguidas de diabetes mellitus.

A conservação da aptidão funcional pode ter sugestões para a qualidade de vida dos idosos, por estar relacionada com a capacidade do indivíduo se manter ativo na comunidade, desfrutando a sua independência até as idades mais avançadas. Os achados deste estudo sugerem que a prevenção e o domínio das doenças crônicas podem melhorar as atividades e, consequentemente, gerar o bem-estar desta população.

A inatividade corporal está intimamente catalogada à incidência e severidade de um amplo número de doenças crônicas. Assim ficando, o exercício físico torna-se uma das ferramentas clínicas mais formidáveis na promoção de saúde.

As pessoas que lidam com diferentes doenças crônicas têm de instruir-se a conviver com várias barreiras em seu cotidiano, uma vez que a doença estabelece adequação do paciente e dos familiares em diferentes aspectos da vida, e comumente tais mudanças se estendem com o tempo. ${ }^{21}$ 
DOI: 10.18606/2318-1419/amazonia.sci.health.v9n2p2-16 Revista Amazônia Science \& Health 2021, Vol. 9, № 2
BARBOSA, MS. ROCHA, WR. QUEROZ, RL. DIAS, AK. MARKUS, GWS. PEREIRA, RA. COUTO, GBF.

Caracterização de idosos residentes no município de Colméia - TO

Gráfico 06: Percepção do idoso sobre o estímulo da sexualidade em sua vida.

\section{Importância da sexualidade}

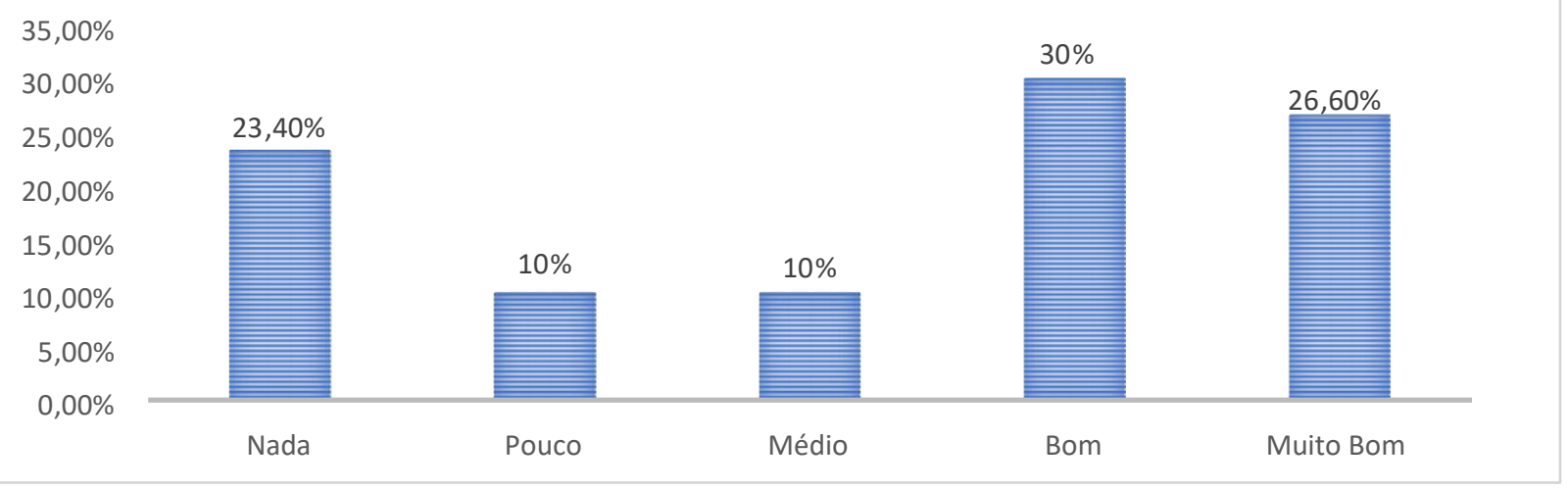

Fonte: Elaborado pelos autores, 2020.

A percepção que a sociedade tem acerca da prática sexual na terceira idade ainda transcorre nos moldes de que a pessoa quando alcança a fase da velhice deixa de ser sexual, adotando a sexualidade. No entanto pode-se observar através dos resultados obtidos através da pesquisa que, uma grande parte de idosos (30\%) consideram a sexualidade como um fator bom, enquanto que $(23,40 \%)$ afirmam que a sexualidade não tem mais nenhuma importância.

A velhice não está ligada a interferência de atividades sexuais, no entanto, ainda é possível encontrar costumes antigos sociais e culturais que acarretam para mitos quando se trata de sexualidade na velhice, e a terceira idade acaba tendo dificuldades de desenvolver de forma integral em sua vida. Ademais a terceira idade encontrasse em constante mudanças, sejam elas físicas, espirituais ou interações familiares levando em consideração que a sexualidade engloba também a qualidade de vida. ${ }^{2}$

[...] não é a idade que determina a ausência do desejo e, muito menos, a ausência ou a presença de relações sexuais mesmo que estas possam ser inscritas na velhice sob tecidos diferentes daqueles encontrados na adolescência e na vida adulta, nos quais computar os orgasmos é uma forma usual. A sexualidade do idoso pode encontrar caminhos inéditos nos quais o desejo, que não morre, encontra outras maneiras de inscrição. ${ }^{13}$

No entanto, o sexo durante a terceira idade ainda envolve bastante preconceito, fazendo com que os idosos evitem manter uma vida sexual ativa, causando consequências 
quanto a percepção de sexo nessa fase da vida. Essa redução dá-se também pelas mudanças fisiológicas ocorridas.

Quando investigados, "como se sentiam ao falar sobre sexualidade", os pesquisadores acreditavam que seria uma dificuldade conseguir trabalhar essa temática, mas, pelo contrário, muitos idosos declararam estar à vontade, relatando ser algo normal em suas vidas.

A influência da cultura sobre a assexualidade dos idosos encontra-se discutida em alguns artigos, preconceitos e tabus estão presentes quando os sujeitos pertencem à faixa etária dos 60 anos. O significado da sexualidade se reduz, em sua maioria, ao órgão genital e ao coito, reduzindo-o para a atividade sexual. Nessa fase da vida, a ausência do companheiro fixo delimita para alguns idosos como o fim das práticas sexuais. A sexualidade não é expressa somente pelo ato sexual e o sexo nem sempre significa penetração, é preciso separar a genitalidade da sexualidade. À medida que o corpo não responde mais ao desejo, as adaptações sexuais se tornam necessárias e ajudam na expressão da sexualidade em idosos. ${ }^{21}$

\section{CONSIDERAÇÕES FINAIS}

Embora a população idosa apresente características em comum, vale mencionar que nem todos os sujeitos que estão nessa faixa etária exibirão os mesmos resultados. Foi possível verificar que a qualidade de vida e o envelhecimento saudável dá-se como uma abrangência que corresponde a uma junção de causas que se relaciona a vivencia diária do idoso, tais como bem estar, autoestima, condicionamento físico e saúde.

Nesse sentido, os assuntos relacionados ao envelhecimento populacional estão envolvidos no contesto social, alinhados aos sistemas de saúdes e de assistência social prestada a esse grupo. Outro fator importante, leva em consideração os cuidados prestados aos membros da terceira idade, tendo em vista que grande parte deles adquirem incapacidades funcionais, o que requer maior cuidado e atenção. Assim, portanto, a incapacidade funcional seja as causadas por: doenças cardiovasculares e cerebrais; Hipertensão Arterial Sistêmica (HAS) e Diabetes Mellitus (DM), são encontradas na pesquisa, entende-se que estudos desse caráter tem a capacidade de fornecer subsídios de utilidade acerca de como os idosos e as famílias no Brasil se adaptam ao envelhecimento. 
DOI: 10.18606/2318-1419/amazonia.sci.health.v9n2p2-16 Revista Amazônia Science \& Health 2021, Vol. 9, № 2
BARBOSA, MS. ROCHA, WR. QUEROZ, RL. DIAS, AK. MARKUS, GWS. PEREIRA, RA. COUTO, GBF.

Caracterização de idosos residentes no município de Colméia - TO

A Organização Mundial da Saúde salienta que a efetividade de políticas voltadas para a prevenção de doenças crônicas não transmissíveis envolve questões de legislação, regulamentação e educação em massa, uma vez que mudanças individuais de comportamentos são complexas de acontecer na carência de mudanças ambientais.

Os resultados aqui apresentados mostraram altas prevalências de doenças crônicas, indicando a necessidade premente de políticas públicas para a redução dessas exposições que podem acometer essa faixa etária da vida, tanto entre idosos quando entre adultos mais jovens.

\section{REFERÉNCIAS}

1.Cancela, DMG. O processo de envelhecimento. Disponível em:http://www.psicologia.pt/pesquisa/index.php?q=diana\%20manuela\%20gomes>.

2. Uchôa YS, Costa DCA, Júnior IAPS, et al. A sexualidade sob o olhar da pessoa idosa. Rev. Bras. Geriatr. Gerontol. 2016; 19(6): 939-49.

3. United Nations. World population prospects: key findings and advance tables. The 2017 re - vision. New York: United Nations; 2017.

4. Pernambuco CS, Rodrigues BM, Bezerra JCP, Carrielo A, Fernandes ADO, Vale RGS, et al. Quality life, elderly and physical activity. Health. 2012 [cited 2017 Mar]; 4 (2):88-93. Available from: http://file.scirp.org/pdf/Health20120200006_92712293.pdf.

5. Cabral M, Ferreira P. O Envelhecimento Ativo em Portugal: Trabalho, Reforma, Lazer e Redes Sociais. Lisboa: Fundação Francisco Manuel dos Santos, 2017.

6. Jacob L. Animação de idosos: Actividades. Porto: Ambar, 2017.

7. Renald ASV. Qualidade de vida do idoso institucionalizado em meio rural (Dissertação de Mestrado em Educação Social não publicada). Escola Superior de Educação do Instituto Politécnico de Bragança, Bragança, 2017.

8. Beard JR, Officer A, De Carvalho IA, Sadana R, Pot AM, Michel JP, et al. The World report on ageing and health: a policy framework for healthy ageing. Lancet. 2016 [acesso em 15 jun. 2017; 387 (10033): 2145-54.

9. Alves JED. A transição demográfica e a janela de oportunidade. São Paulo: Instituto Fernand Braudel de Economia Mundial; 2018.

10. Groisman D. A velhice, entre o normal e o patológico. História, Ciências, Saúde Manguinhos, 61-78, 2017. 
DOI: 10.18606/2318-1419/amazonia.sci.health.v9n2p2-16
BARBOSA, MS. ROCHA, WR. QUEROZ, RL. DIAS, AK. MARKUS, GWS. PEREIRA, RA. COUTO, GBF.

Caracterização de idosos residentes no município de Colméia - TO

11. Sequeira C. Cuidar de idosos com dependência física e mental. Lisboa: lidel - edições técnicas, Lda, 2017.

12. Vasconcelos AMN, Gomes MMF. Transição demográfica: a experiência brasileira. Epidemiol Serv Saúde, 2016; 21 (4): 539-48.

13. Mucida A. O Sujeito não envelhece: psicanálise e velhice. Rio de Janeiro (RJ): Autêntica, 2015.

14. Lopes MJ, Araújo JL, Nascimento EGC. O envelhecimento e a qualidade de vida: a influência das experiências individuais. (2016, abril-junho).

15. Geboers B, Brainard JS, Loke YK, Jansen CJM, Salter C, Reijneveld SA et al. The association of health literacy with adherence in older adults, and its role in interventions: a systematic metareview. BMC Public Health. 2015 [cited 2016 Aug 15]; 15(1):903. Available from: https://bmcpublichealth. biomedcentral.com/track/pdf/10.1186/s12889-015- 2251-y.].

16. UNITED NATIONS. Department of Economic and Social Affairs, Population Division. World population prospects:the 2015 revision, key findings and advance tables. Working Paper ESA/P/WP 241. 2015.

17. Kessler S. Atividade ocupacional, aprendizagem e qualidade de vida em idosos. In: ARGIMON, I. I. L.; ESTEVES, C. S.; WENDT, G. W. (Org.). Ciclo vital: perspectivas contemporâneas em avaliação. Porto Alegre: EDIPUCRS, 2015. p. 256.

18. Torquato R, Massi GAA, Santana AP. Envelhecimento e letramento: a leitura e a escrita na perspectiva de pessoas com mais de 60 anos de idade. Psicol Reflex Crítica 2017; 24 (1): 89-98.

19. World Health Organization (WHO). Noncommunicable diseases prematurely take 16 million lives annually, WHO urges more action (2015). Disponível em: Acesso em: 28 fev. 2015.

20. Organização Pan-Americana da Saúde. Envelhecimento Ativo: uma política de saúde. Brasília: OPAS; 2015.

21. Linhares FMP, Pottes AF, Araújo EC, Menezes EP, Siqueira KA. Percepção de idosos sobre o exercício da sexualidade atendidos no Núcleo de Atenção ao idoso em Recife, Brasil. Rev. enferm. herediana. 2018; 1(2):93-103. 